\title{
Illusion decrement and transfer of illusion decrement in obtuse- and acute-angle variants of the Poggendorff illusion
}

\author{
JOHN PREDEBON \\ University of Sydney, Sydney, New South Wales, Australia
}

\begin{abstract}
Illusion-decrement and transfer-of-illusion-decrement procedures were used to examine the contribution of the obtuse- and acute-angle components of the Poggendorff pattern to the standard Poggendorff illusion. In the first four experiments, subjects were required to scan between the oblique lines of the Poggendorff pattern during the inspection phase of the decrement procedure. However, because of a possible confound associated with this procedure, a different decrement technique was used in Experiment 5. The results of Experiment 5 confirm and extend MacKay and Newbigging's (1977) finding that similar amounts of transfer to the standard pattern are obtained from the obtuse- and acute-angle patterns as from the standard pattern itself: In showing that the acute- and obtuse-angle components both contribute to the illusion, these findings question the plausibility of those theories of the Poggendorff illusion which do not assign any significant role to the acute-angle component. Furthermore, the potential confound associated with the decrement procedure of Experiments 1-4 suggests that the results of other studies obtained with similar procedures need to be reevaluated.
\end{abstract}

The classical form of the Poggendorff illusion refers to the apparent misalignment of two objectively collinear oblique lines separated by a pair of vertical lines, as in Figure 1A.

Although it is frequently classified as an illusion of direction, several investigators (Day \& Dickinson, 1976; Greist-Bousquet \& Schiffman, 1981b, 1985; Pressey, 1971; Quina-Holland, 1977; Zanuttini, 1976) have suggested that the Poggendorff misalignment may be due, at least in part, to the apparent contraction of the distance between the parallels. Recently, Greist-Bousquet and Schiffman (1981b, 1985), following the early work of Judd (1899), proposed that the underestimation of interparallel extent is caused by the relative influence of the amputated forms of the wings-out and wings-in Müller-Lyer figure contained within the Poggendorff figure, as shown in Figures $1 \mathrm{~B}$ and $1 \mathrm{C}$ with the transversals and in Figures 1D and 1E without the transversals, respectively. According to Greist-Bousquet and Schiffman (1985), although wings-out overestimation is greater than the wings-in underestimation for conventional full-line Müller-Lyer figures, the reverse applies for the amputated Müller-Lyer figures. Specifically, as indicated by the results of Greist-Bousquet and Schiffman (1981c) and Warren and Bashford (1977), amputated wings-in Müller-

Thanks are extended to Peter Wenderoth for his comments on a draft of this paper, and to two anonymous reviewers for their helpful critical reviews of the manuscript. Correspondence should be addressed to John Predebon, Department of Psychology, University of Sydney, Sydney, NSW 2006, Australia.
Lyer figures produce strong underestimation effects, whereas amputated wings-out figures promote weak overestimation effects. Thus, in the case of the Poggendorff figure, the net effect of the amputated wings-out (Figure 1D) and wings-in (Figure 1E) Müller-Lyer components is to produce an underestimation of the intertransversal extent and, as a consequence, an apparent misalignment of the oblique lines.

Greist-Bousquet and Schiffman's proposal has been investigated by assessing whether or not the magnitude and direction of the misalignment effect in variants of the classical Poggendorff figure are associated with predictable covariations of the apparent intertransversal extent. GreistBousquet and Schiffman (1981b) found intertransversal underestimation in all Poggendorff variants that showed the classical Poggendorff effect and no distortion in figures in which the misalignment was either small or absent. Similarly, manipulations of the components of the Poggendorff figure produce distortions of intertransversal extent similar to the length distortions found with comparable manipulations of the components of the Müller-Lyer figure (Greist-Bousquet \& Schiffman, 1985). Although the results of these two studies are consistent with the Müller-Lyer theory of the Poggendorff illusion, there is also disconfirming evidence. For example, Finlay and Caelli (1975) and Wilson and Pressey (1976) reported overestimation of interparallel extent. Recently, Trueman and Wilson (1989) found that apparent alignment judgments and judgments of intertransversal distance did not follow similar trends, and others (Day, Jolly, \& Duffy, 1987; Wenderoth \& O'Connor, 1987) also have reported findings at variance with the Müller-Lyer explanation of 


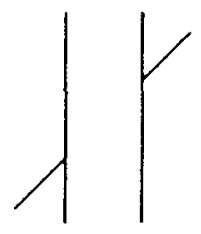

A

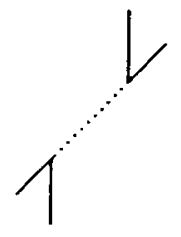

B

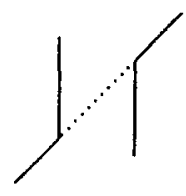

C

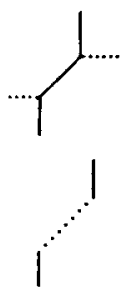

D
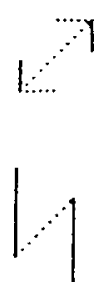

E

Figure 1. The conventional Poggendorff figure (A) contains the wings-out and wings-in Müller-Lyer components shown with (B and $C$, respectively) and without ( $D$ and $E$, respectively) the transversals. The complete Müller-Lyer is shown above Figures $\mathbf{D}$ and $\mathbf{E}$.

the Poggendorff illusion (however, see Greist-Bousquet \& Schiffman, 1987; Greist-Bousquet, Schiffman, Dorsett, \& Davis, 1989).

In the experiments reported here, I used the transferof-illusion-decrement paradigm to examine GreistBousquet and Schiffman's proposal. Illusion decrementthat is, the reduction of illusion magnitude with practice or inspection-occurs with many visual geometric illusions, including the Poggendorff illusion (Beckett, 1989; Cameron \& Steele, 1905; Coren \& Girgus, 1972; MacKay \& Newbigging, 1977; Pressey \& Sweeney, 1969). My aim in the present experiments was to assess whether or not the illusion decrement arising from practice with the wings-in and wings-out Poggendorff variants-hereafter also referred to as obtuse- and acute-angle componentstransfers to the conventional form of the illusion.

Greist-Bousquet and Schiffman do not specify whether their Müller-Lyer theory of the Poggendorff illusion necessarily assumes that the wings-in and wings-out Müller-Lyer components in the Poggendorff figure are distinct illusion types. However, if these two components are indeed distinct illusions, as has been claimed in other contexts (Greist-Bousquet \& Schiffman, 1981a, 1981c), their theory predicts different amounts of transfer from the acute- and obtuse-angle variants to the conventional Poggendorff-illusion figure. In the case of the obtuse-angle variant, practice should fatigue the processes promoting the underestimation of apparent extent and, hence, result in a positive transfer (reduction in illusion magnitude) to the complete pattern. Conversely, in the case of the acuteangle variant, to the extent that the wings-out component produces a weak, or nonexistent, overestimation of apparent extent, practice with this variant should produce either no transfer or a negative transfer (i.e., an increase in illusion magnitude) to the conventional Poggendorff pattern.

Of the few studies carried out to examine illusion decrement in the Poggendorff illusion, only the results of MacKay and Newbigging's (1977) Experiment 4 bear directly on the present investigation. They found that the acute and obtuse patterns shown in Figure 2 produced equivalent amounts of transfer to the Poggendorff pattern.
This finding, however, does not disconfirm the Müller-Lyer theory of the Poggendorff illusion, since both patterns contain the obtuse-angle component. What would be problematic for the theory would be for the Poggendorff variants containing only the obtuse-angle component and only the acute-angle component to produce similar amounts of transfer to the Poggendorff pattern.

In the first four experiments described below, the illusion-decrement procedure was identical to that used by Beckett (1989); this procedure was chosen because Beckett found consistent and substantial decrement of approximately $50 \%$ in the magnitude of the Poggendorff illusion over a 5-min inspection period. However, the results of Experiment 4 suggested a potential confound in Beckett's methodology, and for this reason a different illusion-decrement procedure was used in the last experiment.

\section{EXPERIMENT 1}

In this experiment, the magnitude of the Poggendorff illusion was monitored at 1-min intervals over a 5-min period. The primary issue investigated here was whether or not inspection of the obtuse- and the acute-angle Poggendorff variants differentially affected the magnitude of the Poggendorff illusion over the 5-min inspection period, as might be expected from Greist-Bousquet and Schiffman's apparent distance theory. Two additional conditions were examined: a no-inspection condition to assess the sheer

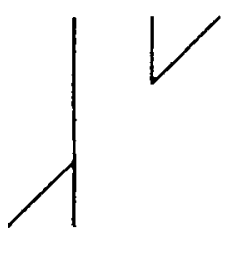

A

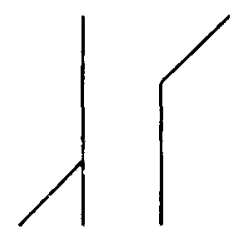

B
Figure 2. Two of the patterns used in MacKay and Newbigging's (1977) Experiment 4: (A) acute, (B) obtuse. 


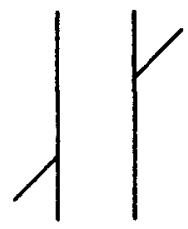

A

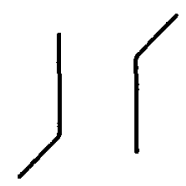

C

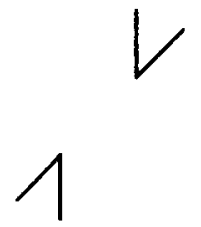

B

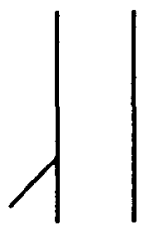

D
Figure 3. Patterns used in Experiment 1: (A) complete, (B) acute, (C) obtuse, (D) standard.

effect of repeated judgments on the magnitude of the Poggendorff illusion, and a control condition in which subjects inspected the conventional Poggendorff pattern.

\section{Method}

Subjects. The subjects were 44 students from an introductory psychology course who were given course credit for their participation in the experiment.

Stimuli. The three inspection patterns and the standard pattern are shown in Figures 3A-3C and Figure 3D, respectively. The patterns were drawn in black ink on separate sheets of white paper. All lines were approximately $1 \mathrm{~mm}$ wide. The 3 -cm-long oblique lines formed an angle of $45^{\circ}$ with the vertical lines, and were physically aligned. The horizontal separation between the vertical lines was $4 \mathrm{~cm}$, and the lengths of the parallel vertical lines were $13.5 \mathrm{~cm}$ for Figures 3A and 3D and $6 \mathrm{~cm}$ for Figures $3 B$ and 3C.

Apparatus. The sheets were presented in a stimulus holder positioned on a table directly in front of the subject. The holder was slanted $70^{\circ}$ away from the subject, and was covered by a $50 \times 60 \mathrm{~cm}$ white cardboard test field containing a 19-cm-diam circular cutout at its center through which the subjects viewed the patterns. When required, a $50 \times 60 \mathrm{~cm}$ white cardboard cover sheet completely $\propto$ cluded the subjects' view of the test field. The seated subject viewed the stimulus sheets from a distance of approximately $45 \mathrm{~cm}$.

Design and Procedure. The subjects were assigned in order of arrival to one of four groups of 11 subjects each. One group did not inspect any pattern during the $1-\mathrm{min}$ intervals between estimates of alignment, while the other three groups inspected one of the three inspection patterns.

The magnitude of the illusion was measured by requiring subjects to mark a point on the right-hand parallel line of the standard figure (Figure 3D) to indicate where the oblique line would appear to terminate if it were extended across the figure. This procedure was illustrated with a Poggendorff figure drawn on a practice card. After the procedure was explained, each subject made an initial, or preinspection, estimate of the apparent position of the oblique line's extension in the standard figure. A 5-min inspection period immediately followed, and during this period the subjects made five more judgments of the standard figure at 1 -min intervals. During the five 1 -min inspection intervals, the subjects in the obruse, acute, and conventional pattern conditions were required to examine the inspection figure carefully by moving their eyes between the two oblique lines; the subjects were monitored to ensure their compliance. In the control condition, a cover sheet occluded the test field during the 1-min inspection intervals, during which time the subjects were instructed to wait until the next judgment.

Because no head restraint was provided, all subjects were cautioned not to tilt their heads. In addition, they were told not to move the marker pen across the figure, although they were permitted to move it along the right-hand vertical line of the test figure.

Each subject's estimate was measured to the nearest $.5 \mathrm{~mm}$ from the point of true collinearity. The data for analyses were the signed error scores, with positive errors indicating the usual Poggendorff effect-that is, the mark was placed below the true point of equality.

\section{Results and Discussion}

The mean errors of the four conditions are shown in Figure 4. A $4 \times 6$ (conditions $\times$ trials) mixed-design analysis of variance (ANOVA) gave a significant main effect of trials $[F(5,200)=14.34, p<.01]$, indicating that illusion magnitude decreased across the trials, and a nonsignificant main effect of condition $[F(3,40)=1.24, p>$ $.05]$. The interaction between trials and condition was significant $[F(5,200)=2.86, p<.05]$, indicating that the four inspection patterns did not produce equivalent amounts of illusion decrement. A comparison of the first and the last trial estimates of alignment confirms this conclusion: Expressed as a percentage of the first estimate, the percentage illusion decrements for the no-inspection, obtuse, acute, and standard figure conditions were $9.3 \%$, $17.6 \%, 21.5 \%$, and $55.6 \%$, respectively.

The main purpose of this experiment was to determine whether the obtuse- and the acute-angle inspection patterns differentially affected the magnitude of the standard Poggendorff illusion over the 5-min inspection period. To evaluate this issue, a $2 \times 6$ (condition $\times$ trials) mixeddesign ANOVA was carried out on these data. The anal-

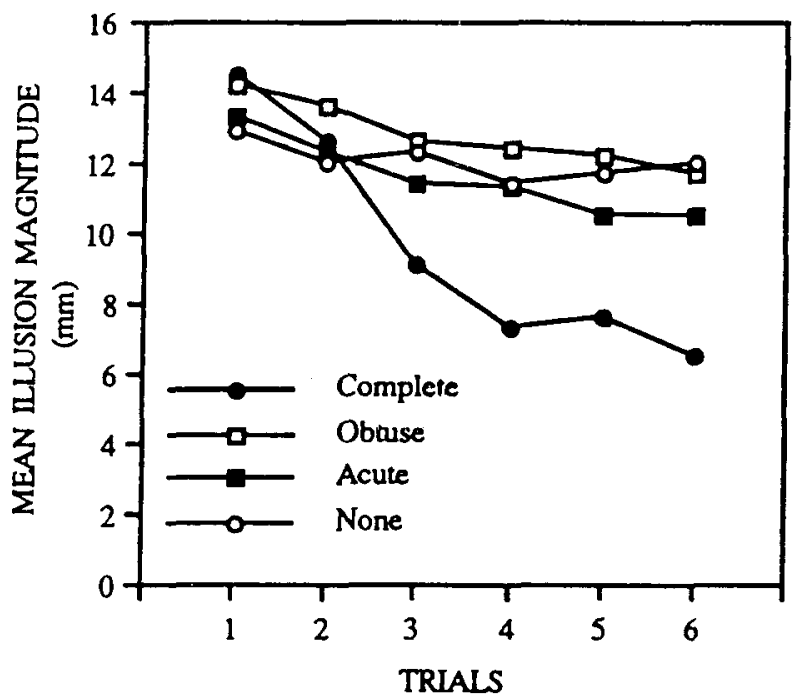

Figure 4. Illusion magnitude (in millimeters) as a function of inspection time for each of the four conditions of Experiment 1: "None" refers to the control condition with no inspection figure between the test settings. 
ysis produced a significant main effect of trials $[F(5,100)=3.02, p<.05]$. However, neither the main effect of pattern nor the trial $\times$ condition interaction was significant $[F(1,20)=.53, p>.05$, and $F(5,100)=.04$, $p>.05$, respectively], indicating that the obtuse and acute inspection patterns produced equivalent amounts of illusion decrement in the standard Poggendorff illusion.

Finally, as a means of describing the data, separate trend analyses were carried out on the data of the four conditions. There were significant linear trends for the data of the complete pattern and for the acute and obtuse patterns $[F(1,50)=70.49,10.41,5.05$, respectively, $p<.05]$ but not for the no-inspection condition $[F(1,50)=3.37$, $p<.05]$. The only other significant trend was the quadratic component for the data of the complete pattern condition $[F(1,50)=6.6, p<.05]$.

The outcomes of this experiment appear to be inconsistent with Greist-Bousquet and Schiffman's theory of the Poggendorff illusion: Rather than either not affecting or increasing the magnitude of the Poggendorff illusion over the five inspection trials, the acute pattern produced illusion decrement equal to that produced by the obtuse pattern. It might be argued, however, that I have misconstrued their theory; rather than independent mechanisms mediating the wings-in and wings-out Müller-Lyer components of the Poggendorff illusion, they might be subserved by a common mechanism. Whether or not this version of their theory can plausibly account for the illusion decrement found with acute-angle patterns is discussed later in conjunction with the results of Experiment 5.

The failure of the acute and obtuse patterns to produce different amounts of transfer might be attributed to aspects of the somewhat unusual decrement procedure used in these two conditions. In the illusion-decrement paradigm, measurements of the magnitude of an illusion are made while the same illusion-inducing pattern is being inspected, whereas in the typical transfer-of-decrement paradigm, measurements of the amount of transfer to a noninspected pattern are made after a variant of the noninspected pattern has been inspected for an extended time period, typically $5 \mathrm{~min}$ or more. The procedure of the complete pattern condition qualifies as an illusiondecrement paradigm (near identity of the standard and inspection patterns). The substantial decrement obtained in this condition is to be expected insofar as it merely confirms the results of other workers (Beckett, 1989; Coren \& Girgus, 1972), who have reported similar amounts of decrement during a 5-min inspection period. In contrast, the decrement procedure of the obtuse- and acute-anglepattern conditions (different standard and inspection patterns) qualifies as an unusual variant of the typical transfer-of-decrement procedure-that is, as five successive 1-min transfer-of-decrement sessions. Unfortunately, because it is not known whether such short sessions yield detectable transfer effects, there could be a number of reasons for the failure to obtain the expected pattern of results in the obtuse and acute pattern conditions. For example, the frequent exposures to the standard pattern during the inspection period may have disrupted or interfered with the processes normally affected by the inspection pattern; or the 1-min inspection periods may have weakly affected the process(es) aroused by the inspection pattern and, consequently, produced a nondetectable effect on the process activated by the standard pattern. Of course, the significant amount of illusion decrement that did occur in the acute pattern condition requires explanation. This decrement cannot be attributed to the effect of repeated judgments of the standard figure, since the no-inspection condition did not produce any significant trends. Perhaps it reflects a general improvement across the inspection trials in a subject's ability to perform the alignment task, as a consequence of the active and prolonged scrutiny of the oblique lines in the inspection patterns.

\section{EXPERIMENT 2}

As noted above, the results of the obtuse- and acuteangle conditions arguably might be attributed to aspects of the somewhat unusual transfer-of-decrement procedure used in these two conditions. Experiment 2 was therefore carried out to determine whether or not similar results would be obtained with traditional illusion-decrement and transfer-of-illusion-decrement procedures. Because of the constraints of the method used to measure illusion magnitude, it was not possible to use identical test and inspection figures. However, the test pattern used to monitor illusion decrement during the 5-min inspection period was similar to the inspection pattern in that it contained only a single obtuse- or acute-angle component for the obtuse and acute inspection patterns, respectively. Transfer of illusion decrement from the Poggendorff variants to the standard Poggendorff pattern was assessed by obtaining pre- and postinspection alignment estimates of the standard Poggendorff pattern.

\section{Method}

Subjects. There were 22 subjects, none of whom had participated in Experiment 1. They were randomly assigned to either the acute or the obtuse pattern condition, with 11 subjects in each one.

Stimuli. The stimuli used in this experiment are shown in Figure 5. The stimuli in Figures 5A-5C were identical to those used in Experiment 1, and those in Figures 5D-5E had identical analogous dimensions.

Procedure. All subjects were first required to make a single estimate of the apparent position of the oblique line in the preinspection standard pattern (Figure 5A). This was followed by a 2-min "rest" period, the purpose of which was to minimize possible carry-over effects from the preinspection pattern on judgments of the subsequently presented test figure. During this period, the subjects performed a visual test. The subjects in the obtuse pattern condition then made an estimate of the apparent position of the oblique line in the obtuse test pattern (Figure 5D), and this was immediately followed by a 5 -min inspection period of the obtuse inspection pattern (Figure 5C), during which five more estimates of the obtuseangle test pattern were made at 1 -min intervals. Following the last estimate of the test pattern, there was another 1-min inspection interval of the obtuse-angle pattern (Figure 5C). At the end of this interval, the inspection pattern was replaced by the postinspection standard pattern (Figure 5A). As in Experiment 1, during the six 1 -min inspection intervals, the subjects were instructed to examine 


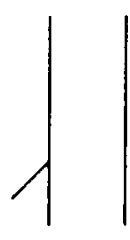

$A$

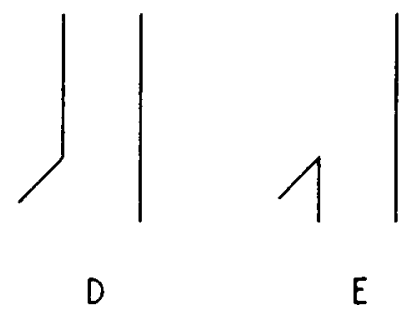

Figure 5. Patterns used in Experiment 2: (A) standard, (B) acute, (C) obtuse, (D) obtuse test, (E) acute test.

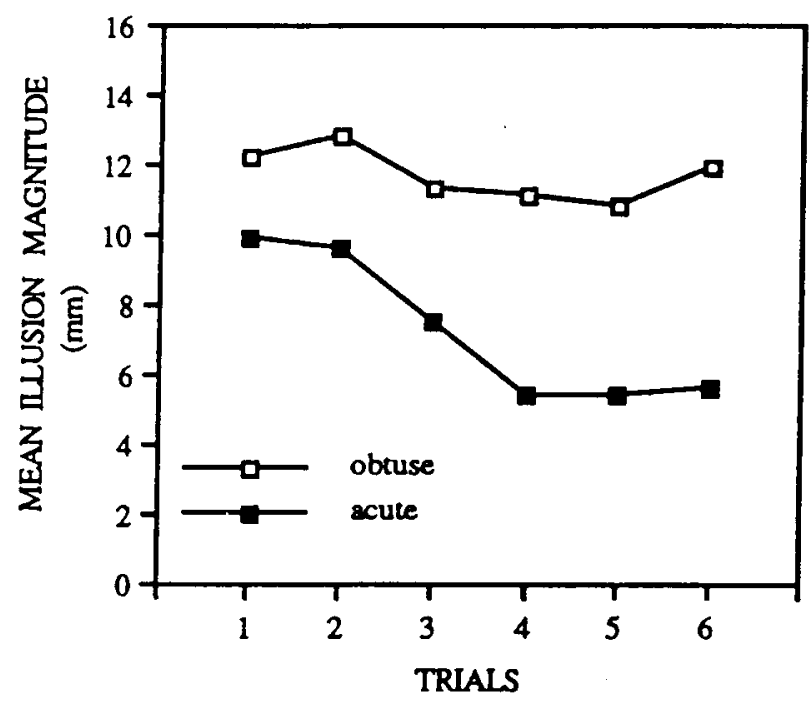

Figure 6. Illusion magnitude (in millimeters) as a function of inspection time for the two conditions of Experiment 2.

the inspection figure carefully by moving their eyes between the two oblique lines. The procedure for the acute-angle condition was identical, except that the inspection and test patterns were the acuteangle variants of the Poggendorff figure (Figures $5 \mathrm{~B}$ and $5 \mathrm{E}$ ).

Each subject provided a total of eight judgments of illusion magnitude-one pre- and one postinspection estimate and six estimates of the test pattern.

\section{Results and Discussion}

The data of the inspection trials are shown in Figure 6. The two-way mixed-design ANOVA on these data showed a significant main effect of pattern $[F(1,20)=6.47$, $p<.05]$ and of trials $[F(5,100)=7.33, p<.05]$ and a significant trial $\times$ pattern interaction $[F(5,100)=3.37$, $p<.05]$. As can be seen in Figure 6, the interaction mainly reflects the reduction in the magnitude of the acute pattern but not the obtuse pattern illusion. Separate trend analyses on the data of the two conditions produced only a significant linear trend component for the data of the acute pattern condition $[F(1,50)=28.11, p<.01]$.

The effect of the inspection period on the magnitude of the noninspected standard Poggendorff figure was assessed by comparing the pre- and postinspection estimates of the standard figure. The means and standard deviations of these data are presented in Table 1. Since illusion decrement during the inspection period occurred only in the acute pattern condition, transfer of the illusion decrement to the standard Poggendorff figure should occur only in this condition. As is evident from Table 1, this is indeed the case: the reduction in the magnitude of the illusion from the pre- to the postinspection period was significant for the acute $[t(10)=5.3, p<.01]$ but not for the obtuse pattern condition $[t(10)=.06, p>.05]$.

The present results indicate that decrement and transfer of decrement to a standard Poggendorff pattern occurs with the acute-angle but not the obtuse-angle inspection pattern. Surprisingly, these findings imply that the obtuse-angle component is not a major determinant of the illusion. However, in view of the results of numerous studies indicating that the obtuse-angle component is an important determinant of the standard Poggendorff illusion, it seemed prudent to suspend judgment on the significance of the present findings pending their independent confirmation. This was the aim in Experiment 3.

\section{EXPERIMENTS 3 AND 4}

Experiment 2 was carried out to determine whether or not illusion decrement in two variants of the Poggendorff figure transfers to the standard Poggendorff illusion. Experiment 3 was carried out to examine the converse casethat is, to determine whether or not illusion decrement in the conventional Poggendorff figure transfers to the acute and obtuse Poggendorff variants.

Experiment 4 was designed to assess the possibility, noted previously, that the moderate amount of decrement that occurred in the acute pattern of Experiment 1 reflected the pattern of eye movements imposed during the inspection phase of the decrement procedure. This was tested by using a parallel-less Poggendorff pattern and monitoring, over the inspection period, the magnitude of the standard Poggendorff illusion.

Table 1

Means and Standard Deviations of Errors in Estimates of Alignment (in Millimeters) for Pre- and Postinspection Standard Poggendorff Figure as a Function of Inspection Condition (Experiment 2)

\begin{tabular}{lccccc}
\hline & \multicolumn{3}{c}{ Inspection Condition } \\
\cline { 2 - 3 } \cline { 5 - 6 } & \multicolumn{3}{c}{ Acute } & & \multicolumn{2}{c}{ Obtuse } \\
\cline { 2 - 3 } \cline { 5 - 6 } & $M$ & $S D$ & & $M$ & $S D$ \\
\hline Pretest & 15.7 & 6.0 & & 14.1 & 5.9 \\
Posttest & 10.2 & 4.9 & & 14.2 & 6.5 \\
\hline
\end{tabular}


Experiments 3 and 4 are reported together for two reasons. First, the two conditions of Experiment 3 and the single condition of Experiment 4 were run together, with subjects randomly allocated to one of the three conditions, at least for the first 5 subjects of each condition. Second, by the time 5 subjects had been tested in all three conditions, it was evident that substantial decrement was occurring with the parallel-less inspection pattern, suggesting a potential confound with the decrement procedure used in Experiments 1-3. It seemed pointless, therefore, to continue running more subjects in Experiment 3. For this reason, there were 5 subjects in each of the two conditions of Experiment 3 and 10 subjects in the single condition of Experiment 4.

\section{Method}

Subjects. A group of 20 subjects who had not participated earlier were tested. They were assigned to the complete (Experiment 3) or parallel-less (Experiment 4) Poggendorff pattern conditions of 10 subjects each.

Stimuli. The stimulus patterns are shown in Figure 7. The dimensions of the patterns were identical to those used in Experiment 1.

Procedure: Experiment 3. The procedure was identical to the transfer-of-decrement paradigm used in Experiment 2. The subjects made a total of eight estimates; there was one pre- and one postinspection estimate of the Poggendorff variant, and there were six estimates of the standard Poggendorff pattern. After a 2-min interval following the preinspection estimate, there was a 5-min inspection of the complete pattern (Figure 7A), during which the subjects made six estimates of the standard pattern (Figure 7D) at 1-min intervals. Following the last estimate of the standard Poggendorff pattern, there was another 1-min inspection of the complete pattern, after which the subjects made a single estimate of the postinspection pattern. For half of the 10 subjects, the pre- and postinspection pattern was the obtuse pattern (Figure 7B); for the other half, it was the acute pattern (Figure 7C).

Procedure: Experiment 4. The procedure was identical to that of the illusion-decrement paradigm of Experiment 1. There were a total of six estimates of the standard Poggendorff pattern

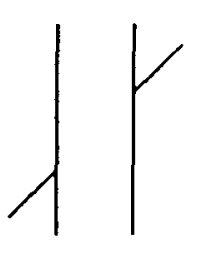

A

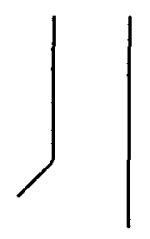

B

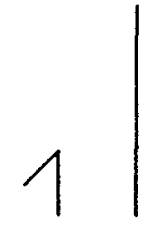

c

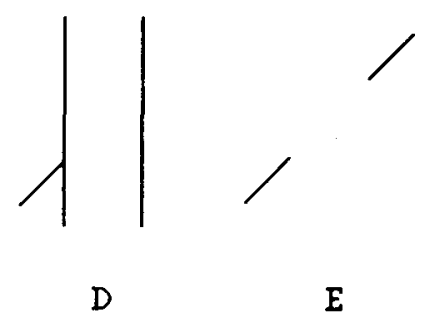

Figure 7. Patterns used in Experiments 3 (A-D) and 4 (D, E): (A) complete, (B) obtuse, (C) acute, (D) standard, (E) parallel-less.

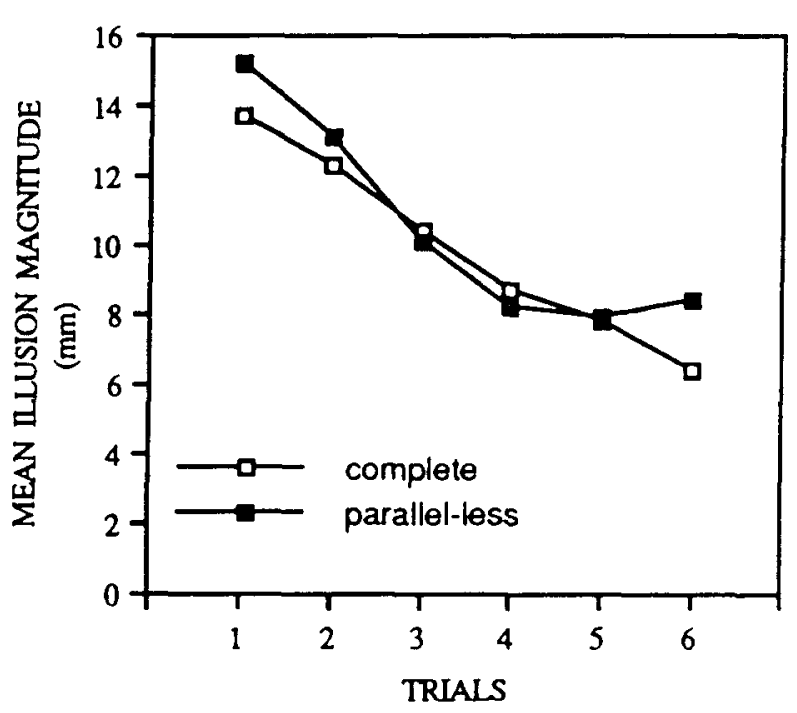

Figure 8. Illusion magnitude (in millimeters) as a function of inspection time for Experiment 3 (complete) and Experiment 4 (parallel-less).

(Figure 7D) made at successive 1 -min intervals. During the five 1-min inspection intervals, the subjects inspected the parallel-less Poggendorff pattern (Figure 7E).

\section{Results}

Experiment 3. A mixed-design ANOVA was performed on the data of the inspection trials to determine whether there were any differences between the two groups of 5 subjects each. The analysis produced a significant main effect of trials $[F(5,40)=15.43, p<.01]$. However, neither the main effect of group $[F(1,8)=4.26$, $p>.05]$ nor the group $\times$ trials interaction $[F(5,40)=$ $.74, p>.05$ ] was significant. These data, therefore, were combined over the group factor, and they are presented in Figure 8 as the complete figure condition. The trend analysis of the combined data produced a significant linear trend component $[F(1,45)=77.59, p<.01]$, indicating that illusion decrement occurred over the inspection period.

To assess whether or not illusion decrement transferred to the postinspection pattern, $t$ tests were performed on the difference between the pre- and postinspection estimates of the noninspected pattern for each of the two subgroups of 5 subjects each. The means of the pre- and the postinspection illusion magnitudes were 12.9 and $8.9 \mathrm{~mm}$, respectively, for the obtuse group, and 9.1 and $4.4 \mathrm{~mm}$, respectively, for the acute group: The difference between the pre- and postinspection means was significant for both the acute and obtuse groups $[t(4)=3.64,2.57, p<.05$, respectively], indicating that illusion decrement transferred to the noninspected acute and obtuse variants of the Poggendorff illusion.

Experiment 4. The results are shown in Figure 8 as the parallel-less figure condition. The one-way repeated measures ANOVA on these data was significant $[F(5,45)$ $=15.88, p<.05]$, and the trend analysis produced a 
significant linear trend component $[F(1,45)=37.41, p<$ $.05]$. These outcomes indicate that the inspection of a pattern containing only the transversals of the conventional Poggendorff figure is sufficient to produce decrement in the standard Poggendorff illusion.

It is clear from Figure 8 that the illusion-decrement functions of Experiments 3 and 4 are very similar. This is confirmed by the results of a $2 \times 6$ (pattern $\times$ trials) mixed-design ANOVA performed on these data. There was a significant effect of trials $[F(5,90)=23.1, p<$ $.05]$, and neither the main effect of group nor the group $\times$ trials interaction was significant $[F(1,18)=.02, p>$ .05 and $F(15,90)=.67, p>.05$, respectively].

\section{Discussion}

The results of Experiment 3 show that illusion decrement in the standard Poggendorff illusion transfers to both the acute- and the obtuse-angle variants. However, interpretation of this finding is complicated by the unexpected results of Experiment 4, indicating that the parallel-less Poggendorff pattern produces almost as much illusion decrement as does the complete Poggendorff pattern. Clearly, since decrement occurs with the parallel-less pattern, the illusion decrement found in some of the conditions of Experiments 1-3 cannot be interpreted unequivocally in terms of the acute- and obtuse-angle components of the inspection pattern.

The spontaneous comments of some of the subjects of Experiment 4 suggested a possible reason for the illusion decrement obtained with the parallel-less pattern; these subjects reported perceiving a white oblique line crossing the vertical parallels of the standard pattern. It is possible, therefore, that the afterimage of the oblique line pattern provided a frame of reference for estimating the apparent position of the oblique line in the standard pattern. Of course, to account for the illusion-decrement function, it must be assumed that the subjects' ability to utilize the afterimage cue improved over the number of trials.

\section{EXPERIMENT 5}

In view of the results of Experiments 3 and 4, in Experiment 5 an illusion-decrement procedure was used to minimize the formation of afterimages of the inspected pattern. The procedure was similar to MacKay and Newbigging's (1977) method for assessing the transfer of illusion decrement from variants of the Poggendorff pattern to the standard Poggendorff figure. Specifically, the method of adjustment was used to obtain estimates of the apparent alignment of the oblique line in the stimulus pattern, and these adjustments were made continuously, during both the illusion-decrement phase and the transfer phase of the experiment. Since the observers were not required to move their eyes in any specified manner, and since the positions of the oblique line component and the adjustment marker were not fixed, it seemed unlikely that afterimages of the oblique line and marker would be formed. ${ }^{1}$

\section{Method}

Subjects. Forty-four undergraduate psychology students participated in partial fulfillment of course requirements. They were randomly assigned to one of four groups of 11 subjects each.

Apparatus. The four stimulus figures are shown in Figure 9. They were presented on a monitor and were generated by an Amiga computer. The lines were approximately $1.5 \mathrm{~mm}$ thick. The vertical lines were $13.5 \mathrm{~cm}$ long and $4 \mathrm{~cm}$ apart. The obliques were $3 \mathrm{~cm}$ long and intercepted the parallels at a $45^{\circ}$ angle.

The computer mouse was used to measure illusion magnitude. Pressing the mouse key caused a 1.4-mm-long horizontal bar marker to appear on a randomly determined point on the right-hand vertical line of the stimulus figures. By moving the mouse, the subject could position the marker at any point on the vertical line. When satisfied with the adjustment of the marker to apparent alignment with the oblique line, the subject released the mouse key; the stimulus figure was then replaced by a dialogue box, and clicking this box initiated the next trial of the series.

The seated subject with head resting in a chin- and headrest viewed the stimuli from a distance of $55 \mathrm{~cm}$, under normal fluorescent room illumination.

Procedure. A single vertical line on the monitor was used to give the subjects practice in the method of adjustment of the marker by mouse movement. When the subjects were satisfied with the adjustment procedure, they were given the test instructions, and the task was illustrated with line drawings of the stimulus figures appropriate to their condition. The instructions required the subjects to adjust the position of the marker to the point on the right-hand vertical line where the oblique line would have appeared to meet it, had it been extended. All subjects then made two pretest alignment estimates of the standard Poggendorff illusion (Figure 9A)one from a starting position of the marker above and one from below the point of physical equality. A 3-min rest interval followed, after which each of the four groups of subjects was then given 24 trials of practice on one of the four patterns shown in Figure 9 and, following a 2-min break, 24 transfer trials on the standard pattern (Figure 9A).

The starting position of the marker was randomly determined by the computer, with the constraint that it be above and below the point of physical equality on alternate trials. There were no time restrictions on making the adjustments, and the subjects were per-

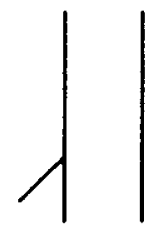

A

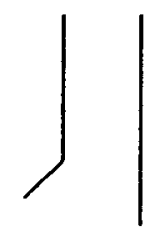

C

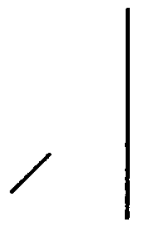

B

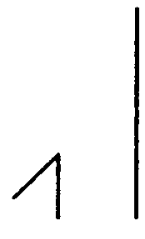

D
Figure 9. Patterns used in Experiment 5: (A) standard, (B) oblique only, (C) obtuse, (D) acute. 
mitted to move the marker in either direction until they were satisfied with their setting. The score was the displacement in millimeters of the marker from the point of physical equality. The usual Poggendorff effect is demonstrated if the marker is set below the point of equality, and these displacements were treated as positive.

\section{Results and Discussion}

The mean of the preinspection estimates of the standard figure for the oblique-only, standard, obtuse, and acute pattern groups were $10.3,11.1,12.6$, and $11.3 \mathrm{~mm}$, respectively: These means are not significantly different from each other $[F(3,40)=.88, p>.05]$.

The results for both the practice and the transfer trials are shown in Figure 10 in blocks of four trials. The $4 \times 6$ mixed-design ANOVA on the practice data showed a significant effect of trials $[F(5,200)=6.70, p<.01]$, a significant effect of groups $[F(3,40)=9.98, p<.01]$, and a nonsignificant group $\times$ trials interaction $[F(5,200)=$ $.77, p>05]$. Although the latter finding suggests that practice had equivalent effects in all four conditions, it is evident from Figure 10 that, unlike the other three practice patterns, the oblique only pattern produced negligible changes in illusion magnitude. The trend analysis performed on each of the four groups confirms this impression. The linear trend component was significant for the standard pattern $[F(1,50)=17.68, p<.01]$, the acute $[F(1,50)=7.43, p<.01]$, and the obtuse $[F(1,50)=$ $9.32, p<.01]$ conditions, but not for the oblique only pattern $[F(1,50)=1.06, p>.05]$.

The ANOVA performed on the transfer data revealed a significant decrement of the illusion over the six blocks

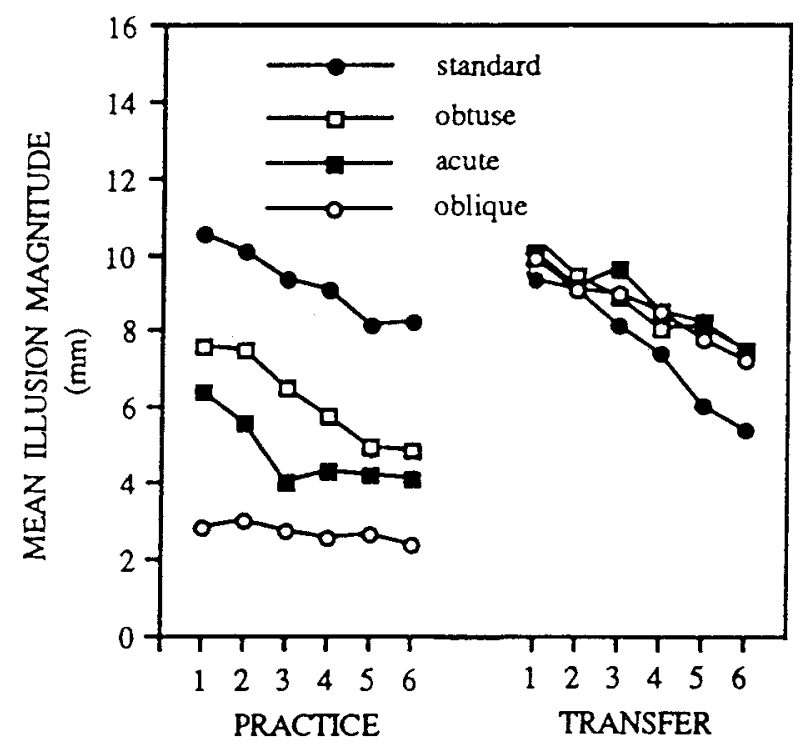

TRIALS

Figure 10. Mean illusion magnitude for the practice and transfer sessions as a function of six blocks of four trials each. The practice trials show the data for each of the four practice patterns (standard, acute, obtuse, oblique only), and the transfer trials show the data of the standard pattern following practice on each of the patterns. of transfer trials $[F(5,200)=22.26, p<.01]$. However, neither the main effect of group $[F(3,40)=.27, p>.05]$ nor the group $\times$ trials interaction $[F(5,200)=.79$, $p>.05]$ was significant, indicating that the type of pattern presented during the practice session produced equivalent effects on the subsequently presented standard pattern. Trend analyses on the transfer data indicated a significant linear trend component for the standard, obtuse, acute, and oblique-only pattern conditions $[F(1,50)=$ $46.53,21.88,26.48,18.79$, respectively, $p<.01$ ]. None of the higher trend components was significant.

To determine whether or not the illusion decrement found in the practice session transferred to the standard Poggendorff figure, $t$ tests were performed on the difference between the means of the preinspection estimates of the standard figure and the mean of the first block of four transfer trials for each group. The difference was significant for the acute, obtuse, and standard patterns $[t(10)=2.96,2.75,2.30, p<.05$, respectively] but not for the oblique only pattern $[t(10)=.64, p>.05]$, indicating that practice with the acute, obtuse, and standard patterns but not with the oblique only pattern significantly reduced the magnitude of the standard figure illusion.

The outcomes of this experiment show that practice with acute and obtuse Poggendorff variants produces effects on the magnitude of a subsequently presented standard form of the Poggendorff illusion that are similar to the effects produced by practice on the standard illusion figure. These findings, then, extend the conclusions of MacKay and Newbigging (1977) to practice patterns containing only a single angle component.

Two additional aspects of the present findings require comment. First, there was an almost complete recovery of the standard Poggendorff illusion during the 2-min break between the practice and transfer sessions. I have no explanation for this phenomenon, apart from noting that MacKay and Newbigging (1977) also reported substantial recovery of the illusion-although in their case, there was a 5-min interval between the practice and test sessions. Second, in view of the similar transfer effects of the practice patterns on the magnitude of the standard Poggendorff illusion, it might be argued that the decrement found in the practice session merely reflects an improvement in the subjects' discriminative ability in performing the alignment task, rather than an effect on illusion magnitude per se. This seems unlikely, however; if it were the case, the recovery of the standard illusion during the break between the practice and transfer sessions would be difficult to understand.

\section{GENERAL DISCUSSION}

The principal aim in the present investigation was to examine Greist-Bousquet and Schiffman's apparent distance theory of the Poggendorff illusion by using illusiondecrement and transfer-of-decrement procedures. The major findings of relevance to their theory may be summarized as follows. Experiment 1, which had a somewhat 
unorthodox transfer-of-training procedure, showed that acute- and obtuse-angle components produced similar amounts of illusion transfer to the standard pattern over the five 1-min transfer-of-decrement sessions. Experiment 2, which had a traditional illusion-decrement procedure, resulted in both illusion decrement and transfer of illusion decrement to the standard Poggendorff pattern with the acute pattern but not with the obtuse pattern. Experiment 3 yielded almost equal amounts of transfer from the standard Poggendorff illusion to the acute and obtuse Poggendorff variants. Unfortunately, because Experiment 4 suggested a potential confound with the decrementinspection procedures used in Experiments 1-3, the results of these four experiments cannot be interpreted unambiguously with respect to Greist-Bousquet and Schiffman's theory nor any other theory of the Poggendorff illusion. However, Experiment 5, which had a different procedure, showed that the amounts of transfer to the standard pattern that are obtained from the acute- and obtuse-angle patterns are equal to those obtained from the standard pattern itself. According to the logic of the transfer-ofdecrement paradigm, this finding suggests that both the acute- and the obtuse-angle components contribute to the standard Poggendorff illusion.

Ignoring for the moment the findings of Experiments $1-4$, the results of Experiment 5 are inconsistent with Greist-Bousquet and Schiffman's theory; rather than a differential amount of transfer, the acute and obtuse patterns produced equivalent transfer of illusion magnitude to the standard Poggendorff pattern. This conclusion, however, is contingent on one's assuming an independence of the mechanisms subserving the obtuse (wingsin) and acute (wings-out) components of the Poggendorff illusion. Can their theory accommodate the present findings if it is modified in favor of the assumption that the two components arouse the same process? In this event, the equivalent amounts of transfer of illusion decrement found in Experiment 5 and the equivalent amounts of illusion decrement found in Experiment 1 for the acute- and obtuse-angle patterns were to be expected, since both patterns were arousing the same mechanism to an equal extent. There is one major difficulty for this modified version of their theory, however. Specifically, given the alleged negligible contribution of the acute-angle component to the magnitude of the Poggendorff illusion, it might be expected that its effectiveness in arousing the mechanism is less than that of the obtuse-angle component. In this case, the obtuse-angle pattern should show a greater transfer of illusion decrement than the acute-angle pattern does. However, neither in Experiment 5 (see Figure 10) nor in MacKay and Newbigging's (1977, Experiment 4) study was there evidence to suggest that the obtuse pattern relative to the acute pattern produces greater transfer to the standard Poggendorff illusion. Moreover, if the acute- and obtuse-angle components arouse the common mechanism to an equal degree, it seems implausible to assume that the acute-angle component contributes a negligible amount to the magnitude of the standard
Poggendorff illusion. From this perspective, the important issue that must be addressed by the theory is (are) the reason(s) for the prepotency of the obtuse-angle component in producing underestimation of interparallel extent in the standard Poggendorff figure.

Critical to Greist-Bousquet and Schiffman's theory is the assumption that the acute-angle component produces little or no misalignment. Yet this assumption is open to empirical refutation. Although it is true that negligible misalignment in acute-angle variants of the Poggendorff was found in some early studies (e.g., Day, 1973), in subsequent studies (e.g., MacKay \& Newbigging, 1977; Wenderoth \& Johnson, 1981), misalignment errors of equivalent magnitude to obtuse-angle variants of the Poggendorff pattern have been reported. Similarly, in the present experiments, the results of the acute pattern (Figure 9D) of Experiment 5 (see Figure 10) and the first trial of the acute test pattern (Figure 5E) of Experiment 2 (see Figure 4) indicate substantial Poggendorff effects. It might be argued, however, that the acute pattern used in the present experiments (Figures 5E and 9D) does contain an implied single obtuse-angle component: The portion of the right-hand vertical line below the point where the imaginary extension of oblique line would intersect the right-hand vertical line conceivably defines such a component (cf. Figures 1E and 9D). Thus, even though the obtuse-angle component is not demarcated by a transversal segment, it is nonetheless contained in the figure, in which case the misalignment obtained with the acuteangle pattern is consistent with Greist-Bousquet and Schiffman's theory.

Although the decrement results have been interpreted as reflecting changes in the mechanisms that mediate the wings-in and wings-out components, other explanations have not been excluded. For example, a number of researchers (e.g., Porac, 1989; Porac \& Coren, 1985) have argued that illusion decrement represents a form of perceptual learning in which modifications of informationprocessing strategies occur as a consequence of scanning the illusion figure, and that transfer of decrement occurs when the practice and test figures are perceived as similar or share a similar global organization (Beckett, 1989; Porac \& Coren, 1985). Leaving aside the problematic findings of Experiments 1-4, the results of Experiment 5 are consistent with this account of decrement: Decrement occurred in all practice figures as a consequence of the recalibration of information-processing strategies on the basis of corrective feedback from eye movements, and significant transfer of illusion decrement to the standard Poggendorff figure occurred for inspection patterns with similar (the acute, obtuse, and standard figures) but not with dissimilar (obtuse only pattern) global organization as the standard figure.

The unexpected finding of Experiment 4, that decrement in the Poggendorff illusion occurs with inspection of the parallel-less Poggendorff pattern, warrants comment. Although it was suggested that afterimages generated by the systematic scanning of the oblique lines may 
explain this finding, it is not clear whether all the results of Experiments 1-3 can be so explained. Excluding the no-inspection condition of Experiment 1, the inspection pattern in all the other conditions of Experiments 1-3 contained the oblique line components and, consequently, the afterimage explanation would seem to predict illusion decrement in all these conditions. This obviously did not occur. In Experiment 2, for example, decrement occurred with the acute but not with the obtuse pattern. Perhaps the steady scanning required to generate an afterimage was disrupted by characteristics of the global configuration that are specific to the obtuse pattern.

Whatever might be the explanation for the findings of the parallel-less Poggendorff pattern of Experiment 4, it is clear that the inspection procedure of scanning the component oblique lines should be used with caution in studies carried out to investigate illusion decrement and transfer of illusion decrement in Poggendorff figures. Otherwise, the resulting decrement could be attributed wholly or in part to the oblique lines of the Poggendorff pattern. Consider the results of Beckett's (1989) study, from which the inspection procedure of Experiments 1-4 was modeled. He found illusion decrement in both real- and subjectivecontour Poggendorff figures, and transfer of illusion decrement from either figure to the other, suggesting that realand subjective-contour figures are probably processed in a similar manner. However, in view of the results of Experiment 4, this conclusion needs independent confirmation, since Beckett's results may be an artifact of subjects' scanning the oblique lines contained in the real- and subjective-contour Poggendorff inspection patterns.

\section{REFERENCES}

Becketr, P. A. (1969). Illusion decrement and transfer of illusion decrement in real- and subjective-contour Poggendorff figures. Perception \& Psychophysics, 45, 550-556.

Cameron, E. H., \& Steele, W. M. (1905). The Poggendorff illusion. Psychological Review Monograph Supplement, 7 (1, Whole No. 29), 83-111.

Coren, S., \& GirGus, J. S. (1972). Illusion decrement in intersecting line figures. Psychonomic Science, 26, 108-110.

DAY, R. H. (1973). The Poggendorff illusion with obtuse and acute angles. Perception \& Psychophysics, 14, 590-596.

DAY, R. H., \& DickInson, R. G. (1976). The components of the Poggendorff illusion. British Journal of Psychology, 67, 537-552.

DAY, R. H., JOLLY, W. J., \& DuFF, F. M. (1987). No evidence for apparent extent between parallels as the basis of the Poggendorff effect. Perception \& Psychophysics, 42, 561-568.

Finlay, D. C., CaElli, T. M. (1975). The Poggendorff illusion and estimates of transverse extent. Perceptual \& Motor Skills, 41, 142-148.

Greist-Bousquet, S., SChiffman, H., R. (1981a). The many illusions of the Mueller-Lyer: Comparisons of the wings-in and wingsout illusions and manipulations of standard and dot forms. Perception, 10, 147-154.
Greist-Bousquet, S., \& Schiffman, H. R. (1981b). The Poggendorff illusion: An illusion of linear extent? Perception, 10, 155-164.

Greist-Bousquet, S., \& SCHIFman, H. R. (1981c). The role of structural components in the Mueller-Lyer illusion. Perception \& Psychophysics, 30, 505-511.

Greist-Bousquet, S., \& Schiffman, H. R. (1985). Poggendorff and Mueller-Lyer illusions: Common effects. Perception, 14, 427-447.

Greist-Bousquet, S., \& SCHIFrman, H. R. (1987). A note on enhancing the Poggendorff illusion. Perception \& Psychophysics, 42, 202-203.

Greist-Bousquet, S., Schiffman, H. R., Dorsett, D., \& Davis, J. (1989). The relation between apparent extent and alignment in the Poggendorff illusion: A response to "No evidence for apparent extent between parallels as the basis of the Poggendorff effect," by Day, Jolly, and Duffy. Perception \& Psychophysics, 46, 501-504.

JuDD, C. H. (1899). A study of geometrical illusions. Psychological Review, 6, 241-261.

MacKay, D. C., \& Newbigging, P. L. (1977). The Poggendorff and its variants do arouse the same perceptual process. Perception \& Psychophysics, 21, 26-32.

PoRAC, C. (1989). Is visual illusion decrement based on selective adaptation? Perception \& Psychophysics, 46, 279-283.

Porac, C., \& CoRen, S. (1985). Transfer of illusion decrement: The effects of global versus local figural variations. Perception \& Psychophysics, 37, 515-522.

Pressey, A. W. (1971). An extension of assimilation theory to illusions of size, area, and direction. Perception \& Psychophysics, 9, 172-176.

Pressey, A. W., \& Sweeney, O. (1969). A variation of the Poggendorff illusion. Perceptual \& Motor Skills, 28, 883-886.

Quina-Holland, K. (1977). Spatial distortions within the Poggendorff figure and its variants: A parametric analysis. Perception \& Psychophysics, 21, 118-124.

Trueman, M. T., Wilson, A. E. (1989). Examination of apparent extent as an explanation of the Poggendorff effect. Perception \& Psychophysics, 45, 494-500.

WARREN, R. M., \& BASHFORD, J. A. (1977). Mueller-Lyer illusions: Their origin in processes facilitating object recognition. Perception, 6, 615-626.

Wenderoth, P., Johnson, M. (1981). Are the obruse angles the key components of the Poggendorff illusion? Perception, 10, 165-172.

WENDEROTH, P., O'CONNOR, T. (1987). Matching gaps between misaligned pointers demonstrates that lines, both transversal and parallel, enhance Poggendorff illusions. Perception \& Psychophysics, 41, 165-172.

Wilson, A. E., \& Pressey, A. W. (1976). The role of apparent distance in the Poggendorff illusion. Perception \& Psychophysics, 20, 309-316.

Zanuttini, L. (1976). A new explanation for the Poggendorff illusion. Perception \& Psychophysics, 20, 29-32.

\section{NOTE}

1. Another way of overcoming the formation of afterimages is simply to instruct subjects to inspect the pattern. Although such a procedure has been used successfully in the Müller-Lyer decrement literature, it was not adopted here because a pilot study failed to obtain substantial and consistent decrement in the magnitude of the Poggendorff illusion.

(Manuscript received December 27, 1989; revision accepted for publication July 9,1990 .) 